\title{
SMOOTH MUSCLE CELLS AND COLLAGEN FIBRES ARCHITECTURE IN EQUIVOCAL URETEROPELVIC JUNCTION OBSTRUCTION: ELECTRON MICROSCOPY STUDY WITH CLINICAL CORRELATION IN ADULT EGYPTIANS
}

By

\author{
MOHAMED WISHAH $1^{1 *}$, AMR ELKHOLY ${ }^{1}$, MOHAMED H. BADAWY ${ }^{1}$, EHAB \\ HAFIZ ${ }^{2}$, HOSSAM ELGANZOURY ${ }^{1}$, HANI H. NOUR ${ }^{1}$, MOHAMED A. ISMAIL ${ }^{1}$, \\ AHMED S. ZAYED ${ }^{1}$, SAMIR ELDAHSHAN ${ }^{1}$, AHMED A. MEHENA ${ }^{1}$, \\ and TAREK EL-LEITHY ${ }^{1}$ \\ Department of Urology ${ }^{1}$ and Department of Pathology and Electron Microscopy ${ }^{2}$, \\ Theodor Bilharz Research Institute, Embaba P. O. Box 30 Giza, Egypt \\ ('Correspondence: moh.weshahy@gmail.com)
}

\begin{abstract}
Adult patients with ureteropelvic junction obstruction (UPJO) would undergo operative pyeloplasty or considered an equivocal type, the decision for management of equivocal UPJO whether pyeloplasty or watchful follow-up is debatable. Equivocal UPJO referred to findings and patient' symptoms suggested an obstruction, whereas the diuretic renogram is not conclusive to have a decision of pyeloplasty. This study evaluated the smooth muscle arrangement, distribution of collagen fibers, and presence of fibrosis in the UPJ segment in equivocal UPJO and normal UPJ segments using transmission electron microscopy (TEM) methods. The study design consisted of 30 adult Egyptian patients who underwent pyeloplasty for unilateral equivocal UPJO. Ten adult patients who had been operated on for oncological indications constituted the control group. Ten representative specimens of UPJO and 5 representative specimens of the control group were examined by TEM. Clinical study comprised 30 patients having equivocal UPJO and operated upon with pyeloplasty were followed for 2 years. Retrospectively analyses of records of 42 adult patients who had unilateral equivocal UPJO and were on watchful followup for 2 years was done to compare results of Pyeloplasty to watchful follow-up. Electron microscopy study of UPJ segment of equivocal UPJO and the normal control cases showed similar findings with proper arrangement of smooth muscle fibers that were in close contact to each other, without fibroblast imposed between muscle bundles, and few collagen fibers were found. Clinical evaluation of results of pyeloplasty or watchful follow-up in equivocal UOJO in adults showed stable radiological findings, stable split renal function and diuretic renogram. In the watchful follow-up group $12 \%$ of cases required pyeloplasty, indication for pyeloplasty were repeated urinary tract infection, intolerable recurrent pain. The clinical series supported by ultrastructure findings showed that watchful follow-up of equivocal UPJO was an accepted treatment option.
\end{abstract}

Keywords: Ureteropelvic junction obstruction, Hydronephrosis, Pyeloplasty, Ultrastructure.

\section{Introduction}

Equivocal ureteropelvic junction obstruction (UPJO) is the clinical situations where the patient's symptoms are flank pain and is associated with radiological imaging that suggest obstruction where diuretic renogram showed non-definite criteria of obstruction. In a retrospective study on equivocal UPJO that was defined as half-life less than 20 minutes on diuretic renogram, 23 patients were identified and underwent minimally invasive pyelo-plasty, follow-up of these patients showed subjective symptomatic relief and stable kidney function (Ozayar et al,2015).

In prospective study of 27 patients with minimally symptomatic or UPJO and treated conservatively, the UPJO was defined by an obstructive pattern of clearance curve and/ or $\mathrm{T} 1 / 2$ greater than 20 minutes when evaluated with diuretic renogram, follow-up consisted of an office visit and renogram every 6 to 12 months, 15 patients $(71 \%)$ did not need intervention and symptoms were relie- 
ved and patients remained on watchful follow-up with a mean of 48 months. Watchful follow-up was considered failure if there was $10 \%$ loss of relative renal function of the affected kidney, development of pyelonephritis and/or more than 1 episode of acute pain, and pyeloplasty was recommended, the study recommended watchful follow-up for asymptomatic or mildly symptomatic adult patients with UPJO because only approximately $30 \%$ had progression to surgical intervention within 4 years of diagnosis (Gurbus et al, 2011).

Split renal function preoperatively in adult patients with unilateral UPJO may not predict recoverability of renal function following pyeloplasty in adult patients aged $\leq 35$ years (Lixd et al, 2018). Controversies exist on the value of differential renal function (DRF) studies done preoperatively prior to pyeloplasty in adults to predict recoverability of kidney function, patients with preoperative DRF $\geq 30 \%$ showed improvement of DRF postoperatively compared to patients with initial DRF below 30\% ( Ortapamuk et al, 2003). Many theories had been postulated to explain the etiology of UPJO, a study found that UPJO in children the number of interstitial cells of Cajal were absent, or significantly reduced, and that Cajal cells might be responsible for conduction of peristalsis in ureteropelvic junction (UPJ) segment (Senol et al, 2016). Interstitial cells of Cajal are considered the pacemaker cells in the intestine; it was reported to be present in small number in the UPJ. There were few studies of the electrical activities of Cajal cells that support its significance in UPJ milking peristalsis (Lang et al, 2006). There was cumulative evidence of molecular mechanism involved in the etiology of UPJO which may explain the course of UPJO and can be targeted therapeutically and indicate which patient with UPJO will require pyeloplasty or they will improve with conservative treatment (Jackson et al, 2017). Inflammatory cell infiltration of muscle wall (Zhang et al, 2000), different degrees of fibrosis, collagen deposition (Murakumo et al, 1997) and odd muscle fiber arrangement and both muscular hypertrophy and/or hyperplasia were detected in the UPJO (Kaneto et al, 1991; Hosgor et al, 2005).

Smooth muscle atrophy/hypoplasia was reported alongside depletion of nerves to the muscular layer (Murakumo et al, 1997). The findings were noted when the PUJ segment was excised at pyeloplasty and therefore represent late features of PUJO. Ultrastructural evaluation of UPJ segments that were removed during pyeloplasty examined under electron microscopy showed fibrosis and marked alternation of several smooth muscle cells with disorganization of muscle filament pattern and reduction of cell-to-cell junction (Rizzo et al, 1981). Under electron microscopy, ultrastructure of pediatric UPJO was showed excessive collagen fibers between and around the muscle cells and presence of group of compromised muscle cells proximal to the collagenous UPJ segment (Hanna et al, 1976). Activation of apoptosis and alteration of cytoskeleton of UPJ segment might explain the altered function and increased apoptosis in smooth muscle cells in UPJO (Cutroneo et al, 2011).

This study aimed to evaluate the smooth muscle arrangement, distribution of collagen fibers, and the presence of fibrosis in the UPJ segment in equivocal UPJO and normal UPJ segments using TEM methods.

\section{Material and Methods}

Patients: The study design consisted of 72 adult Egyptian patients with the equivocal UPJO, and 10 patients who underwent nephrectomy for renal cell carcinoma, their surgical specimen of UPJ segments were examined under

TEM to represent control group: All patients gave informed consent on the type of management for their UPJO and the use of their clinical data and surgical specimen for research purpose, the study was in accordance of Helsinki declaration. Thirty adult patients underwent Anderson-Hynes dismembered pyeloplasty for unilateral equivo- 
cal UPJ obstruction without crossing vessels, mean age was $28 \pm 10$ years (22 males, 8 females; age range was 18-35). The retrospectively analyzed records of the 42 adult patient who had unilateral equivocal UPJO without crossing vessels and were on watchful follow-up for 2-3 years, they were $25 \pm 9$ years (29 males, 13 females; age range was 16-34 years). All 72 patients had imaging studies showed dilated pelvicalyceal system, obstructive pattern of the clearance curve and/or T1/2 was greater than 20 minutes when evaluated with diuretic renogram, and split renal function $\geq 30 \%$.

The study was conducted between January 2007 and July 2018; all patients had at the initial diagnosis abdominal ultrasound, noncontrast computerized tomography, diuretic renogram and split renal function, urine analysis, and clinical history for symptoms of pain and febrile urinary tract infection (UTI). Follow-up was done every 6 months for 2 years. Patients on watchful follow-up who developed recurrent febrile UTI, stone formation, or decline of kidney function they were treated with pyeloplasty. The ten UPJ segments representing the 30 pyeloplasty patients were processed and were examined under TEM. Control group that represented normal UPJ segment were ten patients who had been operated on for oncological indications that were renal tumors $>7 \mathrm{~cm}$ located in the periphery of the kidney, the UPJ segment were removed from the surgical specimen to be examined for ultrastructural characteristics. The $10^{\text {th }}$ UPJ segment of patients underwent pyeloplasty and the $10^{\text {th }}$ normal control, were examined by TEM to evaluate smooth muscle cells pattern and degree of deposition of fibroblast, abundance of collagen fibers between muscle cells, architecture pattern of smooth muscles, and smooth muscle bundle-to-smooth muscle bundle contact arrangement.

TEM: The UPJ specimens were immersed fixed in the phosphate buffer containing 4\% paraformaldehyde and $3 \%$ glutaraldehyde, $\mathrm{pH} 7.4,4^{\circ} \mathrm{C}$ for $2 \mathrm{hr}$ at $4{ }^{\circ} \mathrm{C}$ and rinsed in phosphate buffer. Tissue was immersed in $1 \%$ osmium tetroxide for $2 \mathrm{hr}$ at $4^{\circ} \mathrm{C}$, rinsed in distilled water and incubated with uranyl acetate overnight. Tissue was dehydrated in a graded series of ethanol and embedded in Epon epoxy resin. Ultrathin sections were cut with an ultra-microtome and immersed in uranyl acetate and then in lead citrate. The specimens were examined with TEM (FEI/ Philips EM208S Transmission Electron Microscope (FEI Electron Optics BV, Eindhoven, Netherlands, XX) The UPJ smooth muscle configuration and arrangement with distribution of fibroblast were assessed from a systematic random sample.

\section{Results}

Electron microscopic examination of UPJ segment in patients underwent dismembered pyeloplasty showed the proper arrangement of smooth muscle cells with perfect smooth muscle-to-smooth muscle contact, no abundant fibroblast between smooth muscles ce1ls, no atrophy or hypertrophy of smooth muscles. The control group of normal UPJ segment showed ultrastructure architecture like that of UPJ segment of patients (Fig.1)

The 42 patients with equivocal UPJO were on watchful follow-up, five patients $(12 \%)$ underwent pyeloplasty during the 2 years follow-up, 2 of them had frequent symptoms of flank pains and 3 had repeated attacks of UTI, split renal function did not show significant changes between preoperative $\&$ postoperative values. Differential renal function values in patients who underwent pyeloplasty did not showed significant changes or increase in renal function of the renal unit post operatively compared to preoperative data. Patients with equivocal UPJO whom were on watchful-follow-up were followed for 2 years; differential renal function values did not show significant changes increase or decrease in renal function (Fig. 2).

\section{Discussion}

Since Anderson and Hynes described the UPJO and did the operation of dismembered pyeloplasty, over the last 50 years there existed conflicting reports on the etiology of 
UPJO in neonatal, pediatric, and adult age groups. Several authors attributed the UPJO to decreased interstitial cells of Cajal in the UPJ segment (Senol et al, 2016; Lang et al, 2006). This theory require more investigation to give more information of the neurophysiology of UPJ that could lead to UPJO, at present this theory lacked proper evidence. Inflammatory cell infiltration of muscle wall different degrees of fibrosis, collagen deposition, and odd muscle fiber arrangement were detected in PUJ obstruction, both muscular hypertrophy/ hyperplasia and atrophy/ hypoplasia were also reported (Zhang et al, 2000; Murakumo et al, 1997; kaneto et al, 1991; Hosgor et al, 2005).

In the present study of ultrastructural architecture of equivocal UPJO in adults showed proper arrangement of smooth muscle cells with perfect smooth muscle-to-smooth muscle contact and neither abundant fibroblast between smooth muscles, nor atrophy or hypertrophy of smooth muscles. Kaneto et al. (1991); Murakumo et al. (1997); Zhang et al. (2000) and Hosgor et al. (2005) in a small series of pediatric UPJO examined under TEM, reported muscle atrophy and abundant fibroblast and collagen fibers in between muscle cells. These data were contradicted to the present findings; possible explanation would be the relatively large sample size of the present series, which were on the equivocal UPJO in adults. Collagen-tomuscle ratio and increased collagen deposition was postulated to be the pathological changed in the UPJ segment that led to obstruction (Hanna et al, 1976). The study did not find abundance of collagen fiber existing between smooth muscle cells. Previous ultrastructure studies of the UPJO segment were applied to a small number of cased of pediatric UPJO, these studies explained the etiology of UPJO to be due to excessive collagen fibers between and around the muscle cells and presence of compromised group of muscle cells proximal to the collagenous UPJ segment (Hanna et al, 1976). Fibrosis and marked alternation of several smooth muscle cells with disorganization of muscle filament pattern and reduction of cell-to-cell junction (Rizzo et al, 1981), these studies were done on congenital UPJO in pediatric age group, and the present results differed from the previous ones by using ultrastructure applied to adult UPJO. There was cumulative evidence of molecular mechanism involved in the etiology of UPJO, which may explain the course of UPJO and can be targeted therapeutically and would indicate which patient with UPJO will require pyeloplasty or they will improve with conservative treatment (Jackson et al, 2017).

Their study agreed with the present findings that anatomical architecture was not the sole etiology of UPJO in both pediatric and adult types, but shown that the UPJ segment in both obstruction and normal cases were of normal pattern. The present clinical finding that watchful follow-up of adult patients with equivocal UPJO would be a good option to patients rather than pyeloplasty, these finding agreed with previous reports supported this treatment strategy (Gurbus et al, 2011; Ozayar et al, 2015; Lixd et al, 2018). The present study of ultrastructure of UPJ segments in adults with equivocal UPJO in comparison to controls clearly showed that there was normal anatomical architecture with proper cell-to-cell adhesion, no excess fibroblast or collagen fibers.

\section{Conclusion}

The present cases of equivocal UPJ obstruction in adults ultrastructure pattern of smooth muscles in the UPJ segment were similar to that of normal UPJ segment where muscle cells are properly arranged with few fibroblasts in the segment with proper cellto-cell contact, neither fibrosis, hyperplasia, nor atrophy. A clinical series of 42 patients with equivocal UPJO were on watchful follow-up for 2 years, $12 \%$ of them required pyeloplasty.

The clinical results supported with ultrastructure findings showed that watchful follow-up proved to be the proper treatment, indication for pyeloplasty would be compli- 
cated UPJO as repeated urinary tract infection, intolerable recurrent pain, and stone formation.

The study limitation was applied to adult patients and did not include pediatric aged patients.

\section{References}

Cutroneo, G, Arena, S, Anastasi, G, 2011: Altered cytoskeletal structure of smooth muscle cells in ureteropelvic junction obstruction. J. Urol. 185: 2314-9.

Gurbuz, C, Best, SL, Donnally, C, 2011: Intermediate term outcomes associated with the surveillance of ureteropelvic junction obstruction in adults. J. Urol. 185:926-9.

Hanna, MK, Jeffs, RD, Sturgess, JM, 1976: Ureteral structure and ultrastructure: Part II- Congenital ureteropelvic junction obstruction and primary obstructive megaureter. J. Urol. 116: 725-30.

Hosgor, M, Karaca, I, Ulukus, C, 2005: Structural changes of smooth muscle in congenital ureteropelvic junction obstruction. J. Pediatr. Surg. 40: 1632-6.

Jackson, J, Richard, J, Coward, RJ, 2018: The molecular biology of pelvi-ureteric junction obstruction. Pediatr. Nephrol. 33:553-71.

Kaneto, H, Orikasa, S, Chiba, T, Takahashi, T, 1991: Three-D muscular arrangement at the ureteropelvic junction and its changes in congenital hydronephrosis: A stereo-morphometric study. J. Urol. 146:909-14.
Lang, RJ, Tonta, MA, Zoltkowski, BZ, 2006: Pyeloureteric peristalsis: role of atypical smooth muscle cells and interstitial cells of Cajal-like cells as pacemakers. J. Physiol. 576:695-705.

Li, XD, Wu, YP, Wei, Y, 2018: Predictors of recoverability of renal function after pyeloplasty in adults with ureteropelvic junction obstruction. Urol. Int. 100:209-15.

Murakumo, M, Nonomura, K, Yamashita, T, 1997: Structural changes of collagen components and diminution of nerves in congenital ureteropelvic junction obstruction. J. Urol. 157:19638.

Ortapamuk, H, Naldoken, S, Tekdogan, UY, 2003: Differential renal function in the prediction of recovery in adult obstructed kidneys after pyeloplasty. Ann. Nucl. Med. 17:663-8.

Ozayar, A, Friedlander, JI, Shakir, NA, 2015: Equivocal ureteropelvic junction obstruction on diuretic renogram-should minimally invasive pyeloplasty be offered to symptomatic patients? J. Urol. 193:1278-82.

Rizzo, M, Faussone, S, Pellegrini, MS, 1981: Ultrastructural aspects of the pathology of the pyeloureteral junction. Boll. Soc. Ital. Biol. Sper. 57: 568-74.

Senol, C, Onaran, M, Gurocak, S, 2016: Changes in Cajal cell density in ureteropelvic junction obstruction in children. J. Pediatr. Urol. 12, 2:89e1-5.

Zhang, PL, Peters, CA, Rosen, S, 2000: Ureteropelvic junction obstruction: morphological and clinical studies. Pediatr. Nephrol. 14:820-6

\section{Explanation of figures}

Fig 1: Ultrastructural pattern of smooth muscles of Ureteropelvic junction (UPJ) in equivocal UPJ obstruction and in normal UPJ: A) Pattern of smooth muscles of UPJ in equivocal UPJ obstruction, showed proper arrangement of muscle fibers without detectable collagen or fibrosis with, muscle bundles in close contact to each other, without fibroblast in between muscle bundles (Scale $2 \mu \mathrm{m}$ ).

B) Pattern of smooth muscles in normal UPJ showed muscle fibers in good arrangement with perfect smooth muscle-to-smooth muscle contact without collagen fibers intervening in between, smooth muscles arranged in both longitudinal and transverse patterns (scale $5 \mu \mathrm{m}$ ).

Fig 2: A representavive cases of equivocal ureteropelvic junction obstruction in adults; (A \& B) intrvenous urography showed mildly dilated dilated pelvicalyseal system while ureter visualised, diuretic renogram delayed clearance and split renal function within normal; (C \& D) a case of equivocal ureteropelvic jubction obstruction in adult showed massively dilated pelvis while split renal function of both kidney within normal range. 

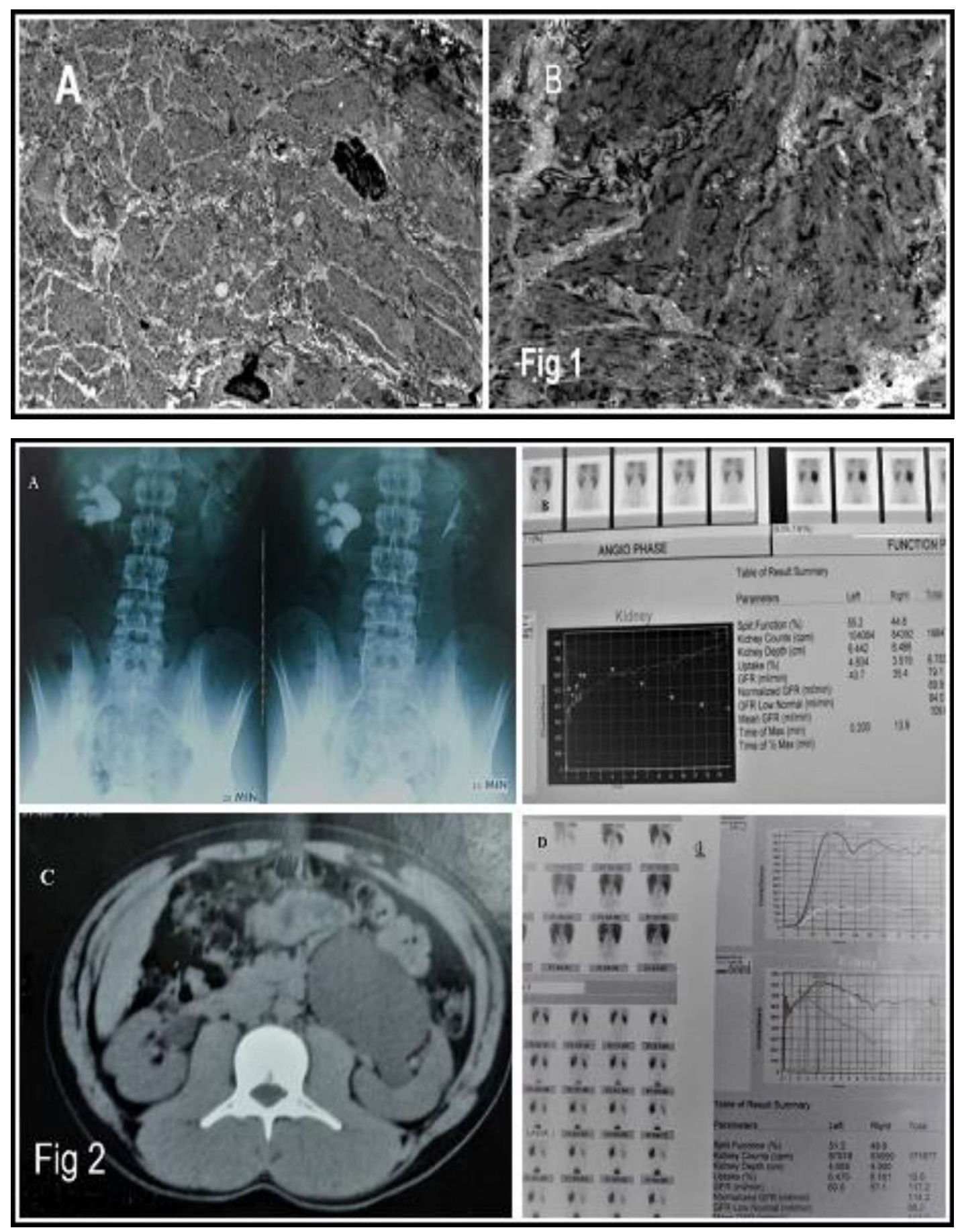